\title{
Coherent X-Ray Radiation Generated by a Divergent Beam of Relativistic Electrons in a Single Crystal in the Beam-Axis Direction
}

\author{
D. O. Shkuropat ${ }^{a}$, S. V. Blazhevich ${ }^{a, *}$, A. S. Gorlov ${ }^{b}$ A. V. Noskov ${ }^{a, b}, * *$, and A. E. Fedoseev ${ }^{a}$ \\ ${ }^{a}$ Belgorod State University, Belgorod, 308015 Russia \\ ${ }^{b}$ Shukhov Belgorod State Technological University, Belgorod, 308012 Russia \\ *e-mail: blazh@bsu.edu.ru \\ **e-mail:noskovbupk@mail.ru
}

Received December 26, 2020; revised January 26, 2021; accepted January 30, 2021

\begin{abstract}
A theory of coherent X-ray radiation generated by a beam of relativistic electrons in a single crystal in the direction close to the electron-beam axis is developed in the Laue scattering geometry. The theory takes into account asymmetry of the electron-field reflection with respect to the crystal surface and the electronbeam divergence. Expressions describing the spectral-angular densities of parametric X-ray radiation near the electron-velocity direction, of transition radiation, and their interference are obtained. The influence of the electron-beam divergence and asymmetry of the electron-field reflection with respect to the target surface on the spectral-angular radiation densities is studied.
\end{abstract}

Keywords: single crystal, parametric X-ray radiation, transition radiation, beam of relativistic electrons

DOI: $10.1134 / \mathrm{S} 1027451021040170$

\section{INTRODUCTION}

Parametric X-ray radiation (PXR) is generated near the Bragg scattering direction because of the scattering of pseudophotons of the relativistic-electron Coulomb field at a system of parallel atomic crystal planes [1-3]. In addition to photons generated in the Bragg scattering direction, radiation theory predicts PXR photon generation near the relativistic-electron velocity direction (forward PXR (FPXR)) [4-6].

The first reports about the discovery of the FPXR of relativistic electrons in a single crystal in the Laue scattering geometry appeared in $[7,8]$. The authors of [8] recorded the X-ray radiation of relativistic electrons from a thick absorbing single-crystal target under FPXR generation conditions in their experiments; however, the sought reflection was manifested rather weakly against the background of radiation produced by electrons at components of the experimental-setup design and also against that of transition radiation (TR) and the possible interference of FPXR and TR. There were no experiments for studying the FPXR properties afterwards. Thus, the theoretical study of the FPXR properties and the search for optimal conditions for clearer experimental observation of the given dynamic effect remains important.
For the case of symmetric reflection in the Laue and Bragg scattering geometries, a detailed theoretical description of FPXR in a single crystal was given in [9, 10]. FPXR theory for the case of asymmetric reflection of the electron field with respect to the target surface where the reflecting planes of the single crystal were located at an arbitrary angle to the target surface was developed in [11]. The influence of the electronbeam divergence on coherent $\mathrm{X}$-ray radiation in the Bragg direction in the Laue scattering geometry was considered in [12]. The theory of coherent $\mathrm{X}$-ray radiation of the beam of relativistic electrons in single crystals in the direction close to that of the beam axis in the Bragg scattering geometry was developed in [13]; and that in periodic layered media, in [14].

In this paper, within the framework of diffraction theory, we obtain and study expressions describing the FPXR spectral-angular characteristics in the Laue scattering geometry for asymmetric reflection in general. We show the influence of the electron-beam divergence on the FPXR angular density. We show the influence of the reflection asymmetry on the spectralangular characteristics of coherent $\mathrm{X}$-ray radiation. 


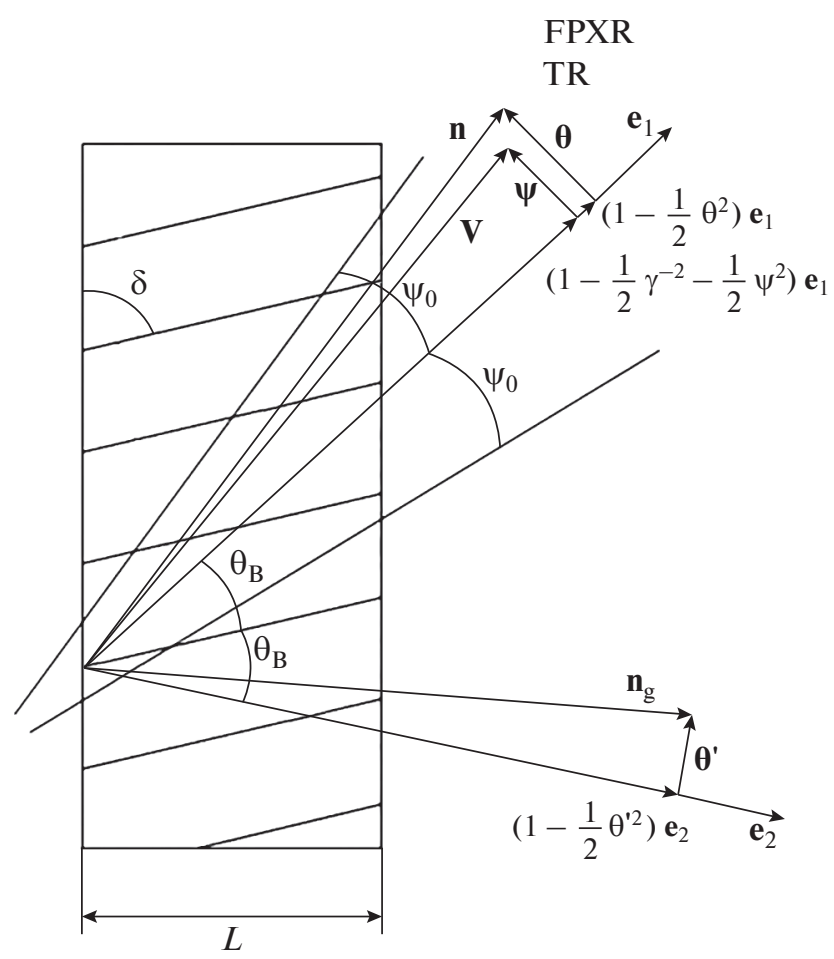

Fig. 1. Geometry of the radiation process.

\section{GEOMETRY OF THE RADIATION PROCESS}

We consider the radiation of a beam of relativistic electrons crossing a single crystal in the Laue scattering geometry (Fig. 1). Let the reflecting system of parallel atomic planes of the single crystal be located at an angle $\delta$ to the target surface (Fig. 1), which corresponds to the case of asymmetric reflection of the radiation field $(\delta=0$ is the particular case of the symmetric reflection). We introduce the angular variables $\boldsymbol{\psi}, \boldsymbol{\theta}^{\prime}$, and $\boldsymbol{\theta}$ in accordance with definitions of the rel- ativistic-electron velocity $\mathbf{V}$ and unit vectors: $\mathbf{n}$ in the direction of the momentum of a photon emitted near the electron-velocity vector and $\mathbf{n}_{\mathrm{g}}$ in the Bragg-scattering direction:

$$
\begin{gathered}
\mathbf{V}=\left(1-\frac{1}{2} \gamma^{-2}-\frac{1}{2} \psi^{2}\right) \mathbf{e}_{1}+\boldsymbol{\psi}, \quad \mathbf{e}_{1} \boldsymbol{\psi}=0, \\
\mathbf{n}=\left(1-\frac{1}{2} \theta^{2}\right) \mathbf{e}_{1}+\boldsymbol{\theta}, \quad \mathbf{e}_{1} \boldsymbol{\theta}=0, \quad \mathbf{e}_{1} \mathbf{e}_{2}=\cos 2 \theta_{B}, \\
\mathbf{n}_{\mathbf{g}}=\left(1-\frac{1}{2} \theta^{\prime 2}\right) \mathbf{e}_{2}+\boldsymbol{\theta}^{\prime}, \quad \mathbf{e}_{2} \boldsymbol{\theta}=0,
\end{gathered}
$$

where $\boldsymbol{\theta}^{\prime}$ is the angle of coherent $\mathrm{X}$-ray radiation (PXR and DTR) in the Bragg-scattering direction, which is measured from the direction $\mathbf{e}_{2}$ of Bragg reflection of the photon incident along the electron-beam axis $\mathbf{e}_{1}$; $\boldsymbol{\psi}$ is the deviation angle of the considered electron in the beam, which is measured from the electron-beam axis $\mathbf{e}_{1} ; \boldsymbol{\theta}$ is the angle of coherent $\mathrm{X}$-ray radiation near the direction of the relativistic-electron velocity (FPXR); and $\gamma$ is the electron Lorentz factor. The angular variables are considered in the form of the sum of components parallel and perpendicular to the figure plane: $\boldsymbol{\theta}=\boldsymbol{\theta}_{\|}+\boldsymbol{\theta}_{\perp}, \boldsymbol{\psi}=\boldsymbol{\psi}_{\|}+\boldsymbol{\psi}_{\perp}$. We consider FPXR in the direction of the vector $\mathbf{n}$ (Fig. 1). We call the angle $\psi_{0}$ the initial divergence of the beam of radiating electrons (Fig. 1). The angle $\psi_{0}$ determines a cone limiting the portion of electrons; outside it, the density of electrons is at least $e$ times smaller than that at the beam axis.

\section{SPECTRAL-ANGULAR RADIATION DENSITIES}

Within the framework of the two-wave approximation of dynamic diffraction theory, in the case of a thin nonabsorbing target, expressions for the spectral-angular densities of FPXR, TR, and their interference were obtained using a method analogous to that in [11]:

$$
\begin{gathered}
\omega \frac{d^{2} N_{\mathrm{FPXR}}^{(s)}}{d \omega d \Omega}=\frac{e^{2}}{\pi^{2}} \frac{\Omega^{(s)^{2}}}{\left(\Delta-\chi_{0}^{\prime}\right)^{2}} R_{\mathrm{FPXR}}^{(s)}, \\
R_{\mathrm{FPXR}}^{(s)}=\frac{1}{\xi^{(s) 2}+\varepsilon} \frac{\sin ^{2}\left(\frac{b^{(s)}}{2}\left(\sigma^{(s)}+\frac{\xi^{(s)}-\sqrt{\xi^{(s) 2}+\varepsilon}}{\varepsilon}\right)\right)}{\left(\sigma^{(s)}+\frac{\xi^{(s)}-\sqrt{\xi^{(s) 2}+\varepsilon}}{\varepsilon}\right)^{2}}, \\
\omega \frac{d^{2} N_{\mathrm{TR}}^{(\mathrm{s})}}{d \omega d \Omega}=\frac{e^{2}}{\pi^{2}} \Omega^{(s)^{2}}\left(\frac{1}{\Delta}-\frac{1}{\Delta-\chi_{0}^{\prime}}\right)^{2} R_{\mathrm{TR}}^{(s)},
\end{gathered}
$$




$$
\begin{aligned}
& R_{\mathrm{TR}}^{(s)}=\left(1-\frac{\xi^{(s)}}{\sqrt{\xi^{(s)^{2}}+\varepsilon}}\right)^{2} \sin ^{2}\left(\frac{b^{(s)}}{2}\left(\sigma^{(s)}+\frac{\xi^{(s)}+\sqrt{\xi^{(s) 2}+\varepsilon}}{\varepsilon}\right)\right) \\
& +\left(1+\frac{\xi^{(s)}}{\sqrt{\xi^{(s)^{2}}+\varepsilon}}\right)^{2} \sin ^{2}\left(\frac{b^{(s)}}{2}\left(\sigma^{(s)}+\frac{\xi^{(s)}-\sqrt{\xi^{(s) 2}+\varepsilon}}{\varepsilon}\right)\right) \\
& +\frac{\varepsilon}{\xi^{(s) 2}+\varepsilon}\left(\cos ^{2}\left(\frac{b^{(s)} \sqrt{\xi^{(s) 2}+\varepsilon}}{\varepsilon}\right)-\cos \left(b^{(s)}\left(\sigma^{(s)}+\frac{\xi^{(s)}}{\varepsilon}\right)\right) \cos \left(b^{(s)}\left(\frac{\sqrt{\xi^{(s) 2}+\varepsilon}}{\varepsilon}\right)\right)\right) \text {, } \\
& \omega \frac{d^{2} N_{\mathrm{INT}}^{(s)}}{d \omega d \Omega}=\frac{e^{2}}{\pi^{2}} \Omega^{(s)^{2}}\left(\frac{1}{\Delta}-\frac{1}{\Delta-\chi_{0}^{\prime}}\right) \frac{1}{\Delta-\chi_{0}^{\prime}} R_{\mathrm{INT}}^{(s)},
\end{aligned}
$$

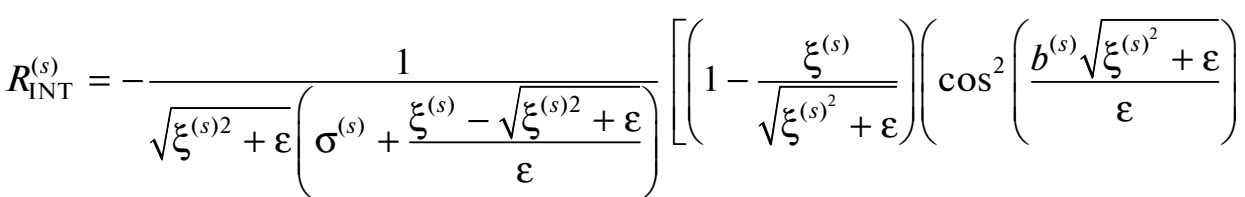

$$
\begin{aligned}
& \left.-\cos \left(\frac{b^{(s)} \sqrt{\xi^{(s) 2}+\varepsilon}}{\varepsilon}\right) \cos \left(b^{(s)}\left(\sigma^{(s)}+\frac{\xi^{(s)}}{\varepsilon}\right)\right)\right)+2\left(1+\frac{\xi^{(s)}}{\sqrt{\xi^{(s)^{2}}+\varepsilon}}\right) \sin ^{2}\left(\frac{b^{(s)}}{2}\left(\sigma^{(s)}+\frac{\xi^{(s)}-\sqrt{\xi^{(s) 2}+\varepsilon}}{\varepsilon}\right)\right),
\end{aligned}
$$

where the following notation is introduced:

$$
\begin{gathered}
\Omega^{(1)}=\theta_{\perp}-\psi_{\perp}, \quad \Omega^{(2)}=\theta_{\|}-\psi_{\|}, \\
\Delta\left(\theta_{\perp}, \theta_{\|}, \psi_{\perp}, \psi_{\|}, \gamma\right)=\gamma^{-2}+\left(\theta_{\perp}-\psi_{\perp}\right)^{2}+\left(\theta_{\|}-\psi_{\|}\right)^{2}, \\
\eta^{(s)}(\omega)=\frac{2 \sin ^{2} \theta_{\mathrm{B}}}{V^{2}\left|\chi_{\mathrm{g}}^{\prime}\right| C^{(s)}}\left(\frac{\omega\left(1-\theta_{\|} \cot \theta_{\mathrm{B}}\right)}{\omega_{\mathrm{B}}}-1\right), \\
\varepsilon=\frac{\sin \left(\delta+\theta_{\mathrm{B}}\right)}{\sin \left(\delta-\theta_{\mathrm{B}}\right)}, \quad b^{(\mathrm{s})}=\frac{1}{2 \sin \left(\delta-\theta_{\mathrm{B}}\right)} \frac{L}{L_{\mathrm{ext}}^{(s)}}, \\
L_{\mathrm{ext}}^{(s)}=\frac{1}{\omega\left|\chi_{\mathrm{g}}^{\prime}\right| C^{(s)}}, \quad v^{(s)}=\frac{\chi_{\mathrm{g}}^{\prime} C^{(s)}}{\chi_{0}^{\prime}}, C^{(1)}=1, \\
\sigma^{(s)}=\frac{1}{\left|\chi_{\mathrm{g}}^{\prime}\right| C^{(s)}}\left(\Delta\left(\theta_{\perp}, \theta_{\|}, \psi_{\perp}, \psi_{\|}, \gamma\right)-\chi_{0}^{\prime}\right) .
\end{gathered}
$$

The functions $R_{\mathrm{FPXR}}^{(s)}, R_{\mathrm{TR}}^{(s)}$, and $R_{\mathrm{INT}}^{(s)}$ describe the FPXR and TR spectral densities and the influence of the FPXR and TR interference on the total spectrum of coherent X-ray radiation. The absence of photoabsorption means that the length of the photon path in the plate $L_{f}$ is significantly smaller than that of X-ray wave absorption in the crystal $L_{a b s}=1 / \omega \chi_{0}^{\prime \prime}$. To reveal dynamic diffraction effects, we consider a single-crystal plate with a thickness such that the length of the electron path in the plate $L_{e} \approx L_{f}=L / \sin \left(\delta-\theta_{\mathrm{B}}\right)$ is many times larger than that of X-ray wave extinction in the single crystal $L_{\text {ext }}^{(s)}=1 / \omega\left|\chi_{\mathrm{g}}^{\prime}\right| C^{(s)}$; i.e., $b^{(s)} \gg 1$. The parameter $\varepsilon$ determines the asymmetry of the electron-field reflection with respect to the target surface.

In expressions (3), $\chi_{\mathrm{g}}^{\prime}$ and $\chi_{\mathrm{g}}^{\prime \prime}$ are defined as follows:

$$
\begin{gathered}
\chi_{\mathrm{g}}^{\prime}=\chi_{0}^{\prime}(F(g) / Z)\left(S(\mathbf{g}) / N_{0}\right) \exp \left(-\frac{1}{2} g^{2} u_{\tau}^{2}\right), \\
\chi_{\mathrm{g}}^{\prime \prime}=\chi_{0}^{\prime \prime} \exp \left(-\frac{1}{2} g^{2} u_{\tau}^{2}\right),
\end{gathered}
$$

where $\chi_{0}=\chi_{0}^{\prime}+i \chi_{0}^{\prime \prime}$ is the average permittivity, $F(g)$ is the form factor of an atom containing $Z$ electrons, $S(\mathbf{g})$ is the structural factor of a unit cell containing $N_{0}$ atoms, and $u_{\tau}$ is the root-mean-square amplitude of thermal vibrations of crystal atoms. In this paper, we consider the $\mathrm{X}$-ray frequency range of $\left(\chi_{\mathrm{g}}^{\prime}<0\right.$, $\left.\chi_{0}^{\prime}<0\right)$. Expression (2) describes $\sigma$-polarized fields at a parameter value of $s=1$ and $\pi$-polarized ones at $s=2$.

We note that expressions (2-4) describe the spectral-angular densities of FPXR and TR and their interference; they were generated by a relativistic electron intersecting a single-crystal plate; the direction of the electron velocity $\mathbf{V}$ and the beam axis $\mathbf{e}_{1}$ form the angle $\psi\left(\psi_{\perp}, \psi_{\|}\right)$.

We consider the spectral-angular densities of the radiation and their interference with the inclusion of the angular divergence of the electron beam $\psi_{0}$. To do this, we perform the averaging of expressions (2-4) over all possible rectangular trajectories of the electron in the beam. As an example, we perform the averaging of the angular FPXR density over the Gaussian distri- 
bution function $f\left(\psi_{\perp}, \psi_{\|}\right)=\frac{1}{\pi \psi_{0}^{2}} e^{-\frac{\psi_{\perp}^{2}+\psi_{\|}^{2}}{\psi_{0}^{2}}}$, and obtain the expressions:

$$
\begin{aligned}
& \left\langle\omega \frac{d^{2} N_{\mathrm{FPXR}}^{(s)}}{d \omega d \Omega}\right\rangle=\frac{e^{2}}{\pi^{2}} \frac{1}{\pi \psi_{0}^{2}} \\
& \times \int_{-\infty}^{\infty} \int_{-\infty}^{\infty}\left(e^{-\frac{\psi_{\perp}^{2}+\psi_{\|}^{2}}{\psi_{0}^{2}}} \frac{\Omega^{(s) 2}}{\left(\Delta-\chi_{0}^{\prime}\right)^{2}} R_{\mathrm{FPXR}}^{(s)}\right) d \psi_{\perp} d \psi_{\|}, \\
& \left\langle\omega \frac{d^{2} N_{\mathrm{TR}}^{(s)}}{d \omega d \Omega}\right\rangle=\frac{e^{2}}{\pi^{2}} \frac{1}{\pi \psi_{0}^{2}} \\
& \times \int_{-\infty}^{\infty} \int_{-\infty}^{\infty}\left(e^{-\frac{\psi_{\perp}^{2}+\psi_{\|}^{2}}{\psi_{0}^{2}}} \Omega^{(s)^{2}}\left(\frac{1}{\Delta}-\frac{1}{\Delta-\chi_{0}^{\prime}}\right)^{2} R_{\mathrm{TR}}^{(s)}\right) d \psi_{\perp} d \psi_{\|}, \\
& \left\langle\omega \frac{d^{2} N_{\mathrm{INT}}^{(s)}}{d \omega d \Omega}\right\rangle=\frac{e^{2}}{\pi^{2}} \frac{1}{\pi \psi_{0}^{2}} \\
& \times \int_{-\infty}^{\infty} \int_{-\infty}^{\infty}\left(e^{-\frac{\psi_{\perp}^{2}+\psi_{\|}^{2}}{\psi_{0}^{2}}} \Omega^{(s)^{2}}\left(\frac{1}{\Delta}-\frac{1}{\Delta-\chi_{0}^{\prime}}\right) \frac{1}{\Delta-\chi_{0}^{\prime}} R_{\mathrm{INT}}^{(s)}\right) \\
& \times d \psi_{\perp} d \psi_{\|} \cdot
\end{aligned}
$$

It is necessary to note that expressions (7-9) are important for determination of the optimal parameters of the experiment for studying the FPXR peak from the point of view of an increase in the intensity of the spectral-angular FPXR density and of optimization of the background of TR and the interference of

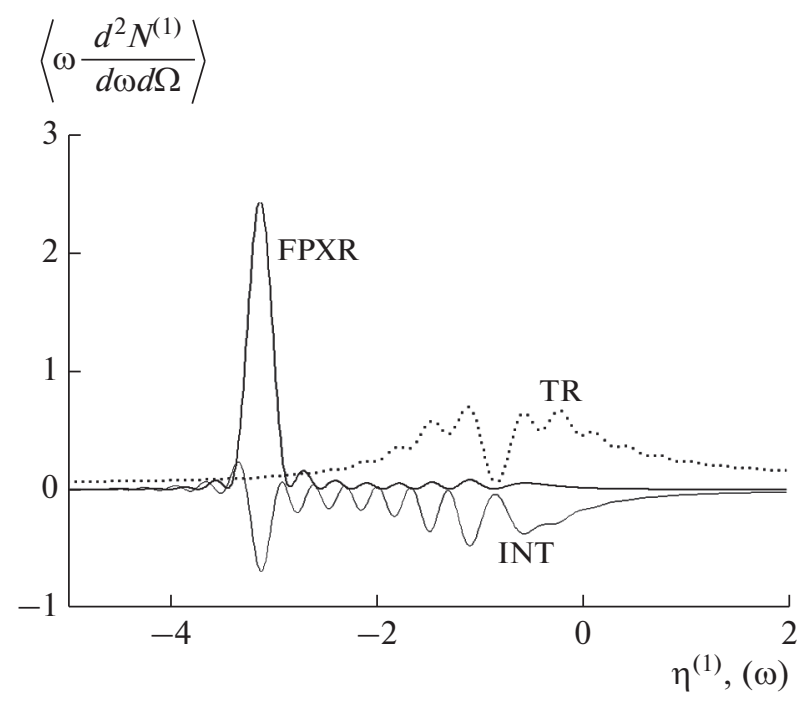

Fig. 2. Spectral-angular densities of FPXR and TR and their interference for a fixed observation angle. An electron-beam divergence of $\psi_{0}=0.1 \mathrm{mrad}$. An asymmetry parameter of $\varepsilon=0.3$.
FPXR and TR. Expressions (7)-(9) can also be used to interpret the results of the performed experiment.

As an example, we numerically calculate the spectral-angular densities of FPXR and TR and their interference for a beam of relativistic electrons intersecting a diamond single-crystal C(111) plate with an energy of $E=102.2 \mathrm{MeV}(\gamma=200)$. We consider the angle between the system of diffracting atomic planes of the single crystal and the axis of the incident electron beam (the Bragg angle) $\theta_{\mathrm{B}}=16.2^{\circ}$, and the Bragg frequency $\omega_{\mathrm{B}}=10.9 \mathrm{keV}$. The system of reflecting (111) planes of the single-crystal target was located at an angle of $\delta=151.3^{\circ}$ to the target surface, which corresponded to the case of electron-field reflection that was asymmetric with respect to the target surface $(\varepsilon=0.3)$. In our calculations, we also considered the angles $\delta=156.4^{\circ}(\varepsilon=0.2)$, and $\delta=138.6^{\circ}(\varepsilon=0.5)$. The target thickness was $L=17.7 \mu \mathrm{m}$. Calculations were carried out for a fixed observation angle corresponding to the maximum of the angular FPXR density $\theta_{\perp}=\sqrt{\gamma^{-2}-\chi_{00}^{\prime}} \approx 6 \mathrm{mrad} ;$ in this case, $\theta_{\|}=0$. We shall carry out our calculations for $\sigma$ polarization $(s=1)$.

Figure 2 shows curves describing the spectralangular densities of FPXR and TR and their interference; they were constructed for a fixed observation angle in accordance with formulas (7)-(9) in the case of an electron-beam divergence of $\psi_{0}=0.1 \mathrm{mrad}$. It is seen that frequencies that are far from the Bragg frequency $\left(\left|\eta^{(s)}(\omega)\right| \gg 0\right)$, are suppressed because of the destructive interference of TR waves emitted from the

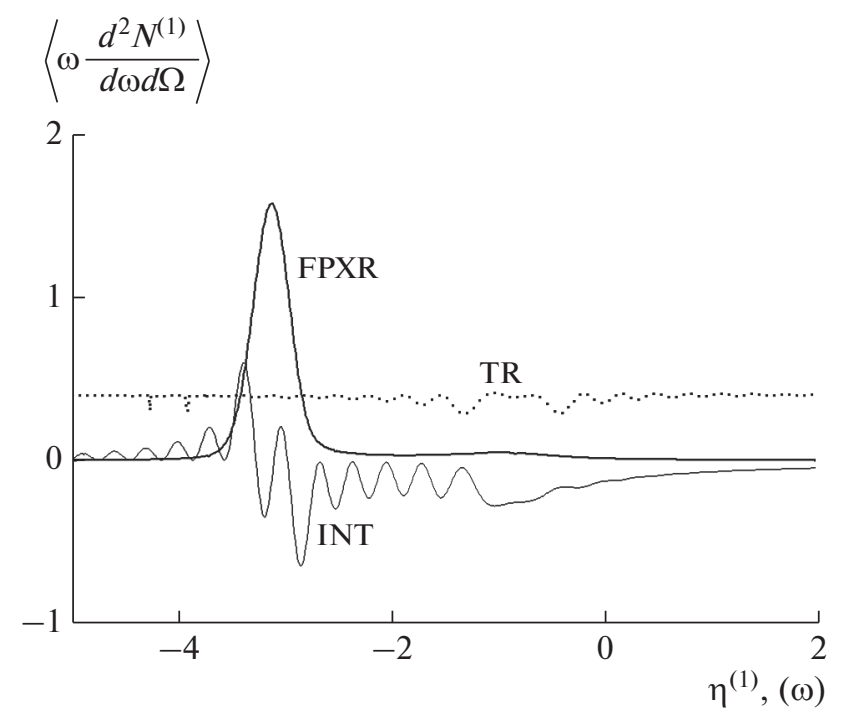

Fig. 3. Spectral-angular densities of FPXR and TR and their interference for a fixed observation angle. An electron-beam divergence of $\psi_{0}=0.5 \mathrm{mrad}$. An asymmetry parameter of $\varepsilon=0.3$. 


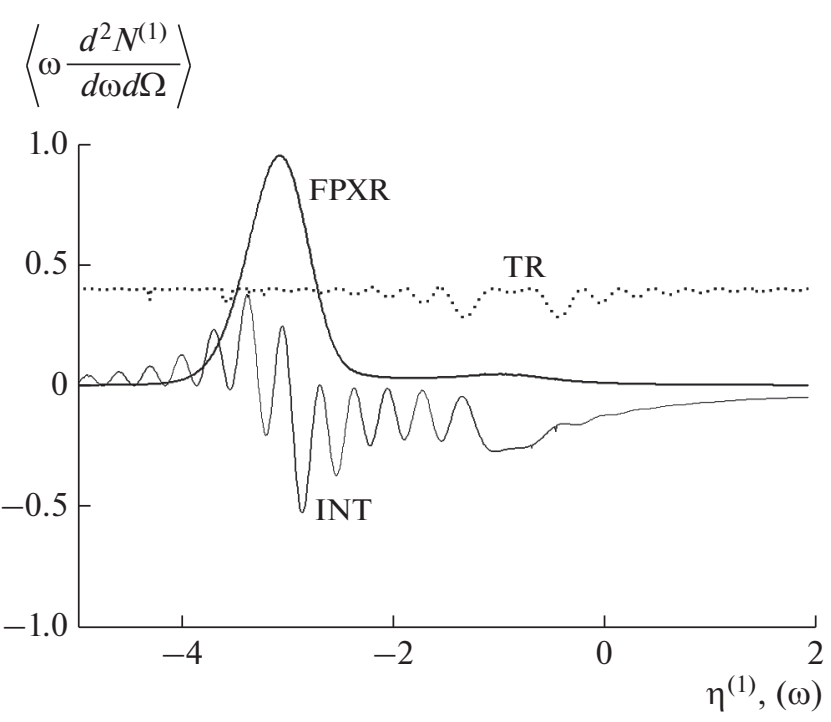

Fig. 4. Spectral-angular densities of FPXR and TR and their interference for a fixed observation angle. An electron-beam divergence of $\psi_{0}=1 \mathrm{mrad}$. An asymmetry parameter of $\varepsilon=0.3$.

input and output target surfaces. This occurred, because the target thickness was chosen such that the resonance condition of the destructive interference of waves with frequencies that are far from the Bragg frequencies was satisfied: $b^{(s)} \sigma^{(s)}=2 \pi m, m$ is the natural number; see the details in [14]. The interference of FPXR and TR affects the FPXR peak destructively in this case. It follows from Fig. 2 that it is possible to experimentally observe the FPXR peak in the sharply asymmetric case of $\varepsilon=0.3$ (symmetric reflection at $\varepsilon=1$ ). We consider the influence of the divergence of the beam of relativistic electrons on the spectral-angular densities of FPXR and TR and on their interference. Figures 3 and 4 shows curves demonstrating the same results as Figure 2, but at $\psi_{0}=0.5 \mathrm{mrad}$ and $\psi_{0}=1 \mathrm{mrad}$, respectively. It follows from Figs. 3 and 4 that, in the case of such electron-beam divergences, TR waves emitted only from the output target surface contribute to coherent $\mathrm{X}$-ray radiation far from the Bragg frequencies $\left(\left|\eta^{(s)}(\omega)\right| \gg 0\right)$. In this case, small oscillations in the TR spectrum are observed in the vicinity of the Bragg frequency; they are related to the interference of waves generated at the input and output target surfaces. Thus, the electron-beam divergence affects the spectral-angular TR density. It also follows from Figs. 3 and 4 that the electron-beam divergence affects the FPXR spectral amplitude noticeably. It is seen that the FPXR spectral amplitude decreases with increasing electron-beam divergence.

We consider the influence of asymmetry of the electron-field reflection with respect to the target surface (the parameter $\varepsilon$ ) on the spectral-angular charac-

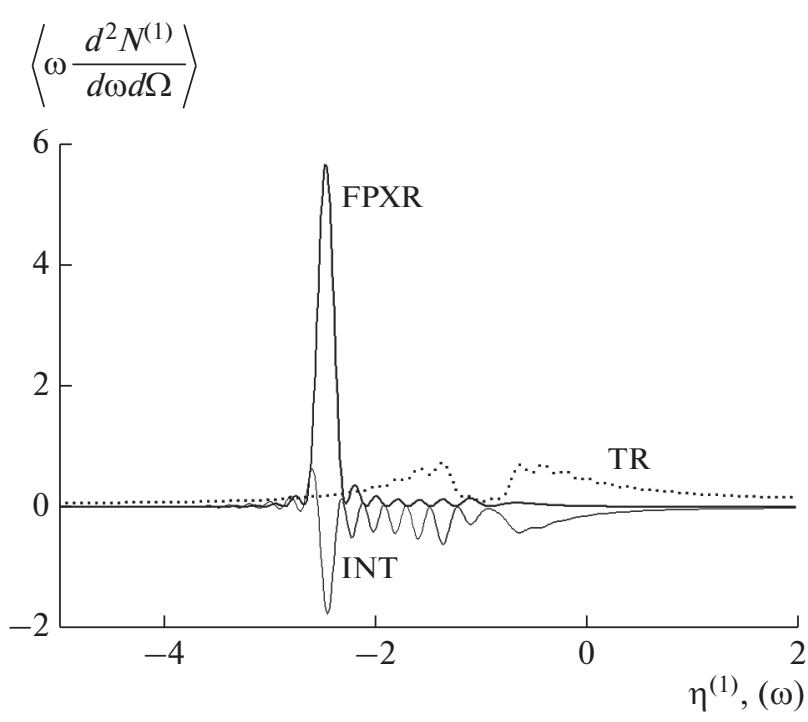

Fig. 5. Spectral-angular densities of FPXR and TR and their interference for a fixed observation angle. An electron-beam divergence of $\psi_{0}=1 \mathrm{mrad}$. An asymmetry parameter of $\varepsilon=0.2$.

teristics of the radiation. In Fig. 2, the spectral-angular characteristics of the radiation are constructed at $\delta=151.3^{\circ}$, which corresponds to $\varepsilon=0.3$. Analogous curves are constructed in Figs. 5 and 6 , but at $\delta=156.4^{\circ}(\varepsilon=0.2)$ and $\delta=138.6^{\circ}(\varepsilon=0.5)$, respectively. In the case of $\varepsilon=0.2$ (Fig. 5), the FPXR spectral amplitude is two times larger than that at $\varepsilon=0.3$; however, the angle $\delta$, changes insignificantly in this case as we see. But, in the case of $\varepsilon=0.5$, on the con-

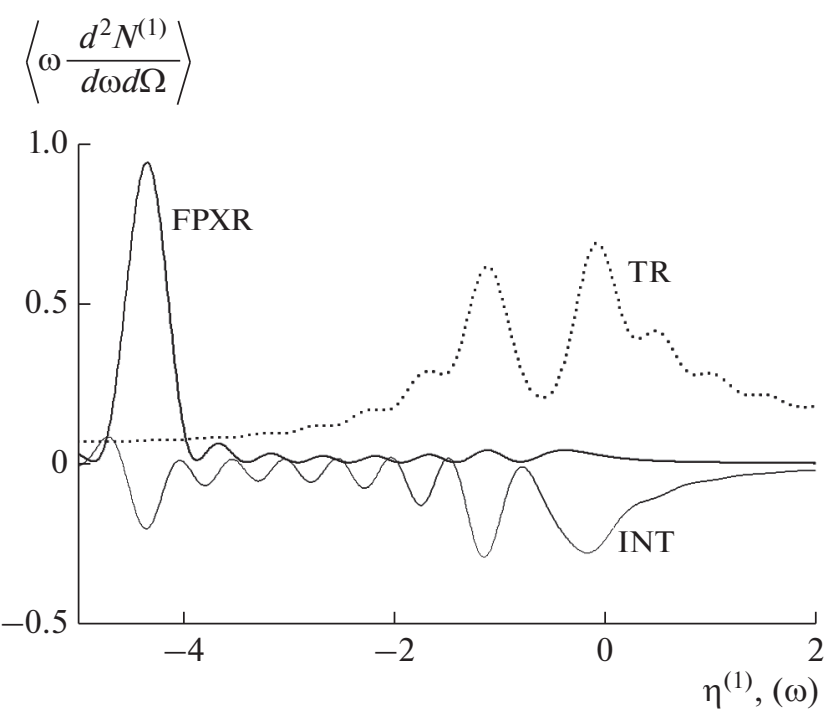

Fig. 6. Spectral-angular densities of FPXR and TR and their interference for a fixed observation angle. An electron-beam divergence of $\psi_{0}=1 \mathrm{mrad}$. An asymmetry parameter of $\varepsilon=0.5$. 
trary, the FPXR spectral amplitude is two times smaller than that at $\varepsilon=0.3$. In this case, the spectralangular TR density changes insignificantly. Thus, the spectral-angular FPXR density depends on the reflection asymmetry significantly. This fact can be used to organize an experiment for seeking and studying the FPXR properties.

\section{CONCLUSIONS}

In the Laue geometry, we have obtained expressions describing the spectral-angular densities of parametric X-ray radiation near the electron-velocity direction, of transition radiation, and their interference. We obtained expressions describing the averaged spectral-angular densities of FPXR, TR, and their interference normalized to one electron as functions of the electron-beam divergence angle $\psi_{0}$ and reflection-asymmetry parameter $\varepsilon$. As an example, we numerically calculated the spectral-angular densities of FPXR, TR, and their interference for a beam of relativistic electrons intersecting a diamond single-crystal plate. We showed that the electron-beam divergence affects the spectral-angular TR density: the FPXR spectral amplitude decreased with increasing electron-beam divergence. We showed that the spectral-angular FPXR density depends significantly on the reflection asymmetry.

\section{REFERENCES}

1. M. L. Ter-Mikaelyan, Effect of the Environment on Electromagnetic Processes at High Energies (Akad. Nauk ArmSSR, Erevan, 1969) [in Russian].
2. G. M. Garibyan and Yan Shi, Zh. Exp. Teor. Fiz. 61, 930 (1971).

3. V. G. Baryshevskii and I. D. Feranchuk, Zh. Exp. Teor. Fiz. 61, 944 (1971).

4. G. M. Garibyan and Yan Shi, Zh. Exp. Teor. Fiz. 63, 1198 (1972).

5. V. G. Baryshevsky and I. D. Feranchuk, Phys. Lett. A 57, 183 (1976).

6. V. G. Baryshevsky and I. D. Feranchuk, J. Phys. 44, 913 (1983).

7. B. N. Kalinin, G. A. Naumenko, D. V. Padalko, et al. Nucl. Instrum. Methods Phys. Res., Sect. B 173, 253 (2001).

8. A. N. Aleinik, A. N. Baldin, E. A. Bogomazova, et al., JETP Lett. 80, 393 (2004).

9. A. S. Kubankin, N. N. Nasonov, V. I. Sergienko, and I. E. Vnukov, Nucl. Instrum. Methods Phys. Res., Sect. B 201, 97 (2003).

10. N. Nasonov and A. Noskov, Nucl. Instrum. Methods Phys. Res., Sect. B 201, 67 (2003).

11. S. V. Blazhevich and A. V. Noskov, J. Surf. Invest.: X-Ray, Synchrotron Neutron Tech. 5, 364 (2010).

12. S. V. Blazhevich and A. V. Noskov, J. Exp. Theor. Phys. 120, 753 (2015).

13. S. V. Blazhevich, K. S. Lyushina, and A. V. Noskov, J. Exp. Theor. Phys. 128, 212 (2019).

14. S. V. Blazhevich, Yu. A. Drygina, O. Yu. Shevchuk, and A. V. Noskov, J. Surf. Invest.: X-Ray, Synchrotron Neutron Tech. 14, 586 (2020).

Translated by L. Kulman

SPELL: 1. OK 